Tasas de cesáreas: análisis de los estimados regionales y nacionales

En 1985, la Organización Mundial de la Salud declaró que no había justificación para que las tasas de cesáreas (porcentaje de partos por cesáreas entre el número total de nacidos vivos) fueran mayores de 10-15\%, independientemente de la región. Dos décadas después, sin embargo, el valor óptimo de la tasa de cesáreas continúa siendo controversial, tanto en los países en desarrollo como en los desarrollados.

En este trabajo se compilaron las tasas de cesáreas por países y se estimaron las tasas regionales con el fin de establecer una línea epidemiológica de base que permita evaluar las necesidades nacionales, regionales y mundiales y desarrollar investigaciones y acciones apropiadas de salud pública. Además, se correlacionaron las tasas de cesáreas con los datos de mortalidad materna, infantil y neonatal y con la proporción de nacimientos atendidos por personal calificado.

Se dispuso de los datos de 126 países, que en su conjunto representan aproximadamente $89 \%$ de los nacimientos ocurridos en todo el mundo en 2002 ( $74 \%$ si se excluyen los datos de China). Las tasas de cesáreas se obtuvieron a partir de encuestas representativas realizadas en los distintos países, de los registros vitales y de las autoridades nacionales.

La tasa estimada de cesáreas en el mundo fue de $15 \%$. La tasa promedio en África fue de 3,5\%, con valores máximos en Sudáfrica $(15,4 \%)$, Egipto $(11,4 \%)$ y Túnez $(8,0 \%)$. El promedio en Asia fue de $15,9 \%$, con las mayores tasas nacionales en China $(40,5 \%)$, Hong Kong $(27,4 \%)$ y Líbano (23,3\%). Nepal y Camboya presentaron las menores tasas $(1,0 \%)$. En Europa, la tasa promedio de cesáreas fue de $19,0 \%$ y los valores máximos se observaron en Italia $(36,0 \%)$ y Portugal $(30,2 \%)$, mientras que los más bajos fueron en Serbia y Montenegro (8,0\%) y Moldova (6,2\%).

Las tasas de cesáreas en América Latina y el Caribe fueron superiores a las de otros países en desarrollo, pero menores que la de los países desarrollados en conjunto. La tasa media fue de $29,2 \%$, con los valores menores en Haití $(1,7 \%)$ y Honduras $(7,9 \%)$ y los mayores en México (39,1\%), Brasil (36,7\%), República Dominicana (31,3\%) y Chile (30,7\%).

Con la excepción de los países latinoamericanos y caribeños, así como de algunos asiáticos, la mayoría de los países con elevadas tasas de mortalidad tenían tasas de cesáreas por debajo del valor recomendado de $10-15 \%$, lo que refleja una fuerte asociación ecológica entre el incremento de las tasas de cesáreas y la disminución de la mortalidad. El conjunto de evidencias presentado en este trabajo respalda la hipótesis de que cuando la tasa de cesáreas sobrepasa mucho el valor de $15 \%$, los riesgos para la salud reproductiva comienzan a sobrepasar los beneficios. El análisis de los resultados indica que las tasas de cesáreas responden ante todo a determinantes económicos: se eleva lentamente en el estrato de bajos ingresos, se incrementa rápidamente en el de ingresos intermedios y vuelve a aumentar lentamente a medida que se alcanza el nivel de saturación con los mayores ingresos. A pesar de que los ingresos y la mortalidad muestran una correlación lineal indiscutible, no hay indicios de que haya una relación inversa entre los ingresos y la tasa de cesáreas, incluso cuando los ingresos son muy elevados.

Las autoridades sanitarias de los países que presentan una mortalidad relativamente alta y tienen tasas de cesáreas muy por encima de los valores de referencia (como Brasil, República Dominicana y México) o donde los indicadores de salud reproductiva ofrecen signos de alarma (como El Salvador, Paraguay y Ecuador) deben investigar si sus sistemas sanitarios están ofreciendo una atención obstétrica adecuada y establecer un sistema de seguimiento de las tasas de cesáreas y de la mortalidad materna.

Este trabajo representa el primer intento de estimar las tasas de cesáreas a nivel mundial y regional y de establecer su posible asociación con indicadores específicos de salud reproductiva. Además, logró conformar la mayor y más representativa serie de datos demográficos y sanitarios de los países en desarrollo (disponible en http://www. measuredhs.com). (Betrán AP, Merialdi M, Lauer JA, Bing-Shun W, Thomas J, Van Look P, et al. Rates of caesarean section: analysis of global, regional and national estimates. Paediatr Perinat Epidemiol. 2007;21:98-113.)

\section{Beneficios de la práctica de ejercicios en mujeres tratadas por cáncer de mama}

Los actuales programas de rehabilitación de pacientes con cáncer se basan principalmente en el apoyo psicoterapéutico o social. Por lo general, 
estas terapias no toman en cuenta los problemas físicos que enfrentan estos pacientes, como la fatiga, la pérdida de capacidad funcional y el aumento de peso. Los ejercicios pueden mejorar una amplia gama de problemas que afectan a la calidad de vida después del diagnóstico de cáncer. Un estudio observacional prospectivo de sobrevivientes de cáncer de mama indicó que el riesgo de muerte se redujo en $50 \%$ en las mujeres que se mantenían activas en comparación con las que permanecieron inactivas después del diagnóstico. Una revisión sistemática reciente sobre el efecto de los ejercicios en las pacientes con cáncer de mama y sobrevivientes de esa enfermedad concluyó que los ejercicios mejoraron la calidad de vida, la capacidad cardiorrespiratoria y el funcionamiento físico y redujeron la fatiga.

En este estudio se evaluó si la participación en un programa supervisado de ejercicios en grupo para mujeres en tratamiento por cáncer de mama en sus primeros estadios ofreció beneficios funcionales y psicológicos durante el tratamiento y si esos beneficios se mantuvieron seis meses después de la intervención.

Se captaron 203 pacientes con cáncer de mama en estadios tempranos (0-III) durante la sesión de asignación de quimioterapia o radioterapia ambulatorias en tres centros oncológicos pertenecientes a los Servicios Nacionales de Salud, en Escocia, Reino Unido. Se excluyeron las pacientes con hipertensión no controlada, enfermedades respiratorias, disfunción cognitiva o que realizaban ejercicios con regularidad. Las pacientes se asignaron aleatoriamente a un grupo de ejercicios o a otro de control. La evaluación se realizó al inicio, a las 12 semanas y a los 6 meses (117 pacientes completaron el seguimiento).

La calidad de vida se estableció mediante la evaluación del tratamiento contra el cáncer (físico, funcional, social y emocional), el nivel de fatiga y los síntomas endocrinos. Como indicador secundario de los resultados se empleó el inventario de depresión de Beck, la escala de afectos positivos y negativos, el índice de masa corporal, el recuento de las actividades físicas en los últimos siete días, el desempeño en la prueba de caminar 12 minutos y la puntuación en la prueba de movilidad de los hombros.

Después de 12 semanas de ejercicios supervisados, el grupo de intervención mostró beneficios en el funcionamiento físico y psicológico en comparación con el grupo de control. No se informaron efectos adversos. Los beneficios informados a las 12 semanas por el grupo que participó en el programa de ejercicios se mantuvieron a los seis meses de seguimiento, con la excepción del tiempo autoinformado de ejercicios físicos. Los beneficios específicos relacionados con la calidad de vida y el cáncer de mama se hicieron evidentes solo a los seis meses, cuando la mayoría de las pacientes habían concluido el tratamiento.

No se logró identificar qué parte del programa de ejercicios generó mayores beneficios. Los datos cualitativos indican que probablemente el efecto de grupo mismo constituyó un aspecto importante. Además, las mejorías en las pruebas de la caminata de 12 minutos y en la de la movilidad de los hombros en el grupo de intervención fueron más atribuibles a los ejercicios en sí que al efecto de grupo.

De esta manera, se concluye que los ejercicios supervisados en grupo, sumados a la atención médica habitual que reciben las mujeres tratadas por cáncer de mama en sus estadios tempranos, ofrecieron beneficios funcionales y psicológicos adicionales. Los médicos deben recomendar a sus pacientes con cáncer de mama mantenerse en actividad y los encargados de diseñar las políticas sanitarias deben ofrecer la posibilidad de practicar ejercicios físicos en los servicios de rehabilitación de pacientes con cáncer, de manera similar a los que se ofrecen para la rehabilitación cardíaca. Se requieren investigaciones adicionales para determinar el efecto de los ejercicios en pacientes con otros tipos de cáncer. (Mutrie N, Campbell AM, Whyte F, McConnachie A, Emslie C, Lee L, et al. Benefits of supervised group exercise programme for women being treated for early stage breast cancer: pragmatic randomised controlled trial. Br Med J. 2007;334:517-22.)

\section{Reducción de la mortalidad infantil en Chile: un éxito en dos etapas}

La mortalidad infantil ha disminuido en la Región de las Américas de 36,9 por 1000 nacidos vivos en el quinquenio 1980-1985 hasta 24,8 por 1000 nacidos vivos en el período 1995-2000. Esta mortalidad, no obstante, se distribuye de forma desigual: mientras en algunos países - como Chile, Costa Rica y Cuba - las tasas están cercanas o por debajo de 10 por 1000 nacidos vivos, en otros - como Bolivia y Haití - se mantienen por encima de 50 por 1000 nacidos vivos. El elemento común en los casos más exitosos ha sido la fuerte intervención estatal, mediante políticas sanitarias estructuradas y sostenidas —enfocadas hacia la salud materno-infantil y el entorno del hogar- y otras políticas sociales relevantes, como la universalización de la educación básica.

En el caso de Chile se observan dos claras etapas en la reducción de la mortalidad infantil. La primera etapa comenzó en la década de 1950, cuando la tasa de mortalidad infantil era de 136,2 por 1000 nacidos vivos (con 29,0 debido a enfermedades infecciosas, diarreas y deshidratación; 44,4 por enfer- 